\title{
COVID-19 Associated Rhino-Orbital-Cerebral Mucormycosis - An Institutional Series
}

\author{
Nishant Rana \\ Associate Professor, Department of ENT, India.
}

Corresponding Author: Nishant Rana, Associate Professor, Department of ENT, India.

Received date: November 01, 2021: Accepted date: December 15, 2021: Published date: January 04, 2022

Citation: Nishant Rana (2022). COVID-19 Associated Rhino-Orbital-Cerebral Mucormycosis - an Institutional series. J. Clinical Otorhinolaryngology, 4(1); DOI:10.31579/2692-9562/041

Copyright: ( $) 2022$, Nishant Rana. This is an open access article distributed under the Creative Commons Attribution License, which permits unrestricted use, distribution, and reproduction in any medium, provided the original work is properly cited.

\section{Abstract}

Invasive fungal infection or mucormycosis is almost always confined to the patients with altered host defenses such as in transplant recipients, diabetics or patients with malignancies. Hypergycemia or uncontrolled diabetes, particularly diabetes acidosis is considered as the strongest and very well known risk factor for mucormycosis. It has spread like fire amongst the active COVID-19 and post COVID-19 diabetic patients. Many studies across the world have established the definitive severity of SARS-CoV-2 infection amongst diabetic patients.

Keywords: cerebral mucormycosis; fungal; hypergycemia

\section{Introduction}

India is recognized as the diabetes and mucormycosis 'capital' of the world [1]. Diabetes is the fastest growing non-communicable disease in India. Coronavirus disease 2019 (COVID-19) second wave in India has presented in a catastrophic way as compared to the first wave and startled the medical fraternity. Severe acute respiratory syndrome Coronavirus 2 (SARS-CoV-2) was found to be associated with systemic immune alterations causing wide range of manifestations including various bacterial and fungal infections [2]. This time India faced a major morbidity and mortality in the form of post COVID-19 sepsis and the abrupt spike of COVID-19 associated mucormycosis (CAM). In the previous 3 years (January 2018 to March 2021) before COVID-19 pandemic we encountered only 5 cases of rhinomaxillary or rhinoorbital-cerebral mucormycosis. COVID-19 infection has led to worst consequences in the patients especially with uncontrolled diabetes, immunocompromised, immunosuppressed (post organ transplant) and those who were managed with long periods or high doses of systemic corticosteroid therapy [3].

Invasive fungal infection or mucormycosis is almost always confined to the patients with altered host defenses such as in transplant recipients, diabetics or patients with malignancies. Hypergycemia or uncontrolled diabetes, particularly diabetes acidosis is considered as the strongest and very well known risk factor for mucormycosis. It has spread like fire amongst the active COVID-19 and post COVID-19 diabetic patients. Many studies across the world have established the definitive severity of SARS-CoV-2 infection amongst diabetic patients [4]. A small number also includes post-transplant (renal transplant) patients, many of which were diabetic also which proved lethal collectively.

Mucormycosis can be classified based on site of involvement as rhinoorbital-cerebral, pulmonary, cutaneous, gastrointestinal and disseminated form [5]. In literature only a limited case series of post COVID-19 mucormycosis have been published [6-11].In this paper we are discussing our institutional experience of a series of 79 cases of rhino-orbitalcerebral mucormycosis related to COVID-19 infection, which to our best knowledge is one of the biggest institutional case series.

\section{Institution series}

In this case series we are retrospectively analyzing the 79 proven cases of COVID-19 related rhino-orbital-cerebral mucormycosis. Throughout the second wave of COVID-19 infection in India, we operated 96 suspected cases of mucormycosis between mid April 2021 and mid September 2021 that also included 7 patients operated elsewhere and referred for further management. Out of all 96 operated cases 79 were histopathologically diagnosed as mucormycosis. These patients presented to us with varied disease load. There were 62 males and 17 females. The youngest patient was 21 years old and eldest one was 72 years old. 15 patients were operated during their active COVID-19 infection whereas rest 64 patients operated during post COVID-19 status after an initial recovery.

All patients had one or other symptoms or signs of sinusitis including facial pain or numbness, facial swelling, headache and nasal congestion. Of these 79 patients of mucormycosis, in 27 patients disease was restricted to nose and paranasal sinuses (PNS) (rhinomucormycosis) [Figure 1], 43 showed orbital involvement as well (rhino-orbital mucormycosis) and 9 patients had severe disease involving brain also (rhino-orbital-cerebral mucormycosis). Hence, total 52 patients $(43+9)$ had orbital involvement. All 52 patients who had orbital involvement had lid edema with proptosis, orbital pain with periocular swelling, conjunctival edema and double vision in varied severity. 


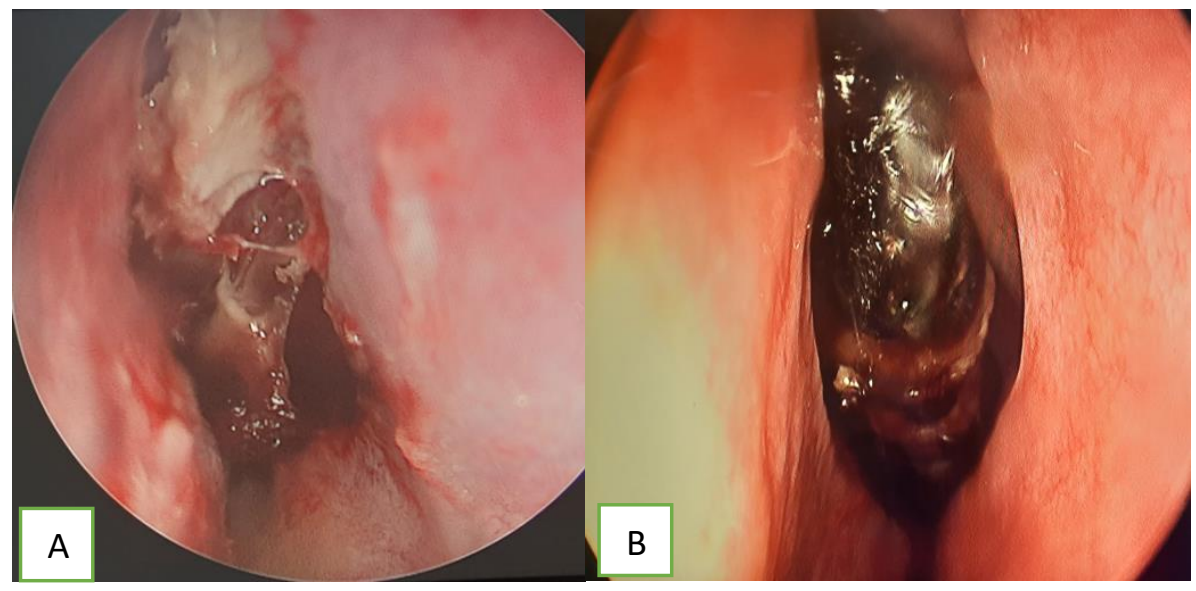

Figure 1: Endoscopic images of (A) necrotic middle turbinate (mucormycosis) and (B) Black turbinate due to rhino-mucormycosis.

11 out of 43 patients of rhino-orbital mucormycosis had positive perception of light (PL) only, 6 of 43 patients had no perception of light with dilated non-reactive pupil (total ophthalmoplegia) whereas rest had just diminished vision of variable grade.[Figure 2] One patient among 6 patient of total ophthalmoplegia had bilateral involvement and 2 also developed pulmonary mucormycosis who died during ICU care.[Figure 3]

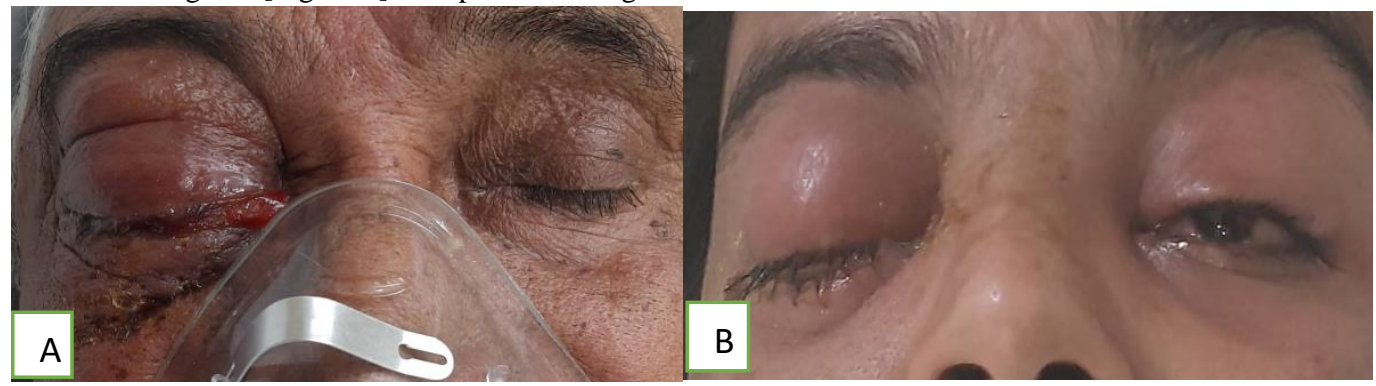

Figure 2: Images showing right orbital involvement due to mucormycosis (total ophthalmoplegia).

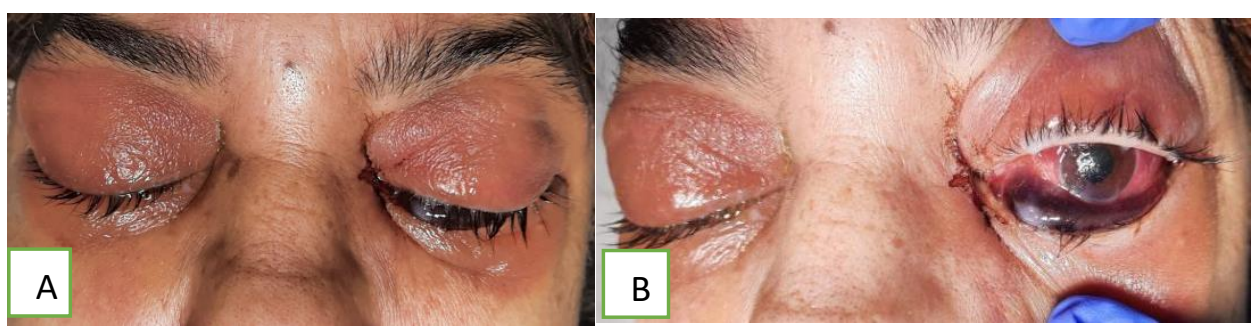

Figure 3: Images showing bilateral orbital involvement due to mucormycosis (A) and non reactive dilated pupil with total ophthalmoplegia (B).

In the 9 patients with cerebral involvement, all patients had altered sensorium, confusion or disorientation of variable grade. 3 out of 9 had unilateral whereas one had bilateral dilated and non-reactive pupil with ophthalmoplegia and rest had diminished or blurred vision of variable grade.

All patients were hyperglycemic during the time of clinical diagnosis or suspicion of mucormycosis. 67 patients had known history of diabetes whereas 12 patients developed steroid induced hyperglycemia. All patients received systemic steroids in the form of oral methylprednisolone, intravenous dexamethasone or intravenous methylprednisolone as a part of management of COVID-19 infection.

Out of total 79 patients, 18 patients had history of ICU care whereas rest 61 patients had non-ICU care. 59 patients required oxygen at some period of COVID-19 infection whereas only 20 patients did not give any history of oxygen requirement.

In all suspected patients, a preliminary nasal swab was sent for $\mathrm{KOH}$ mount, a magnetic resonance imaging (MRI) of paranasal sinuses, orbit and brain was performed to look for extent of the disease if present. Based on preliminary $\mathrm{KOH}$ mount report and MRI findings surgery was planned according to the extent of disease and alongside systemic anti-fungals were also started empirically after the opinion of our infectious disease specialist. Sinusitis was seen in the form of varied level of mucosal thickening of sinuses in all patients. Predominantly unilateral ethmoidal and maxillary sinusitis was seen.

Endoscopic sinus surgery and debridement was performed, all the debrided tissue was sent for histopathology and some tissue with pus if present sent for bacterial and fungal culture. [Figure 4] Thorough local application of amphotericin B $0.1 \%$ ointment was done after debridement and nasal cavities were packed. Out of all 96 operated cases, mucor fungal hyphae with tissue invasion in histopathology was seen in 53 cases; mixed fungal involvement (Mucor, Aspergillus and Candida) in 16 cases; isolated Aspergillus was identified in 10 cases whereas in 17 cases there were no fungal elements identified. But on the basis of clinical and radiological suspicion amphotericin B was started in all 96 patients and doses were monitored and modified after definitive diagnosis by the infectious disease specialist. 


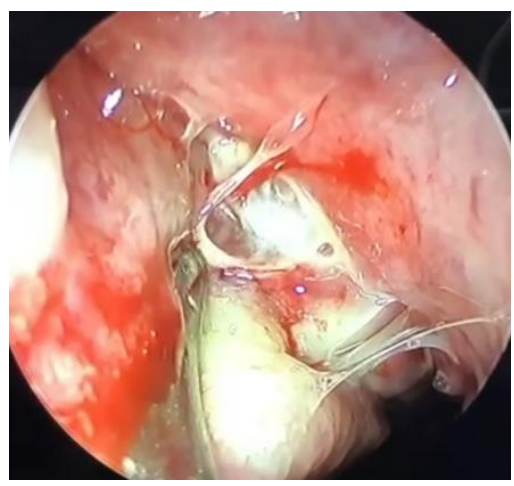

Figure 4: Endoscopic image showing pus draining from maxillary sinus (positive for fungal culture).

In all cases of [(rhino (27), rhino-orbital (43) and rhino-orbital-cerebral (9)] mucormycosis, repeat imaging were performed between $3^{\text {rd }}$ and $7^{\text {th }}$ days post-operatively to see course of disease and to take measures for further debridement if needed. [Figure 5 to 8]

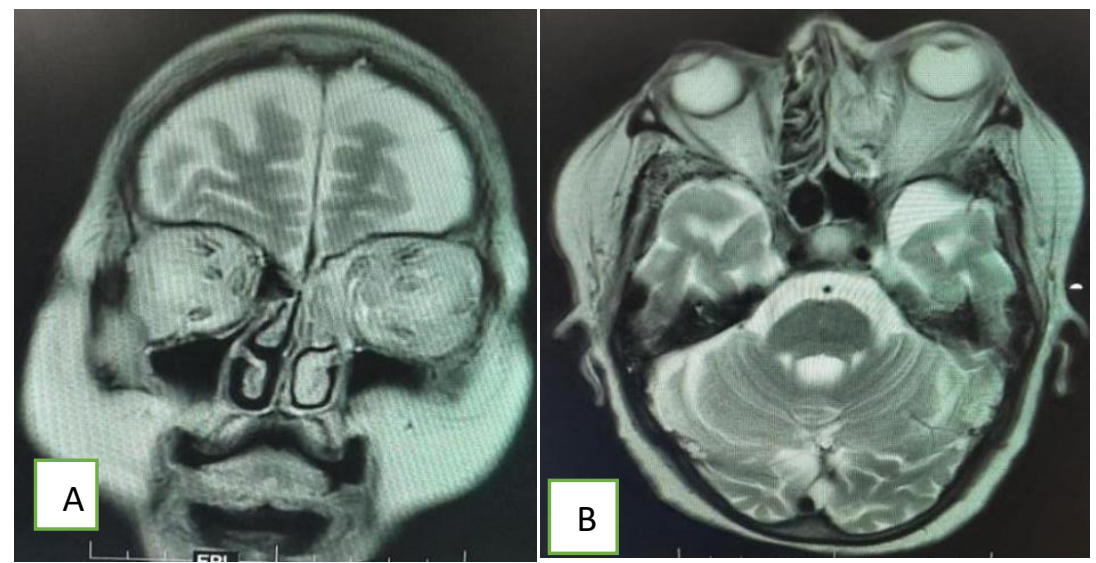

Figure 5: CEMRI (A:coronal; B:axial) of brain and orbits showing features of fungal sinusitis predominantly involving left side ethmoid and frontal sinuses with extension of the disease in intraorbital region through the medial orbital wall with resultant stranding of the retro-orbital and extraconal fat and thickening and hyperintensity of the extraocular muscles. There is proptosis of the left eye. The fat stranding is extending upto the orbital apex with mild asymmetry of the left cavernous sinus.

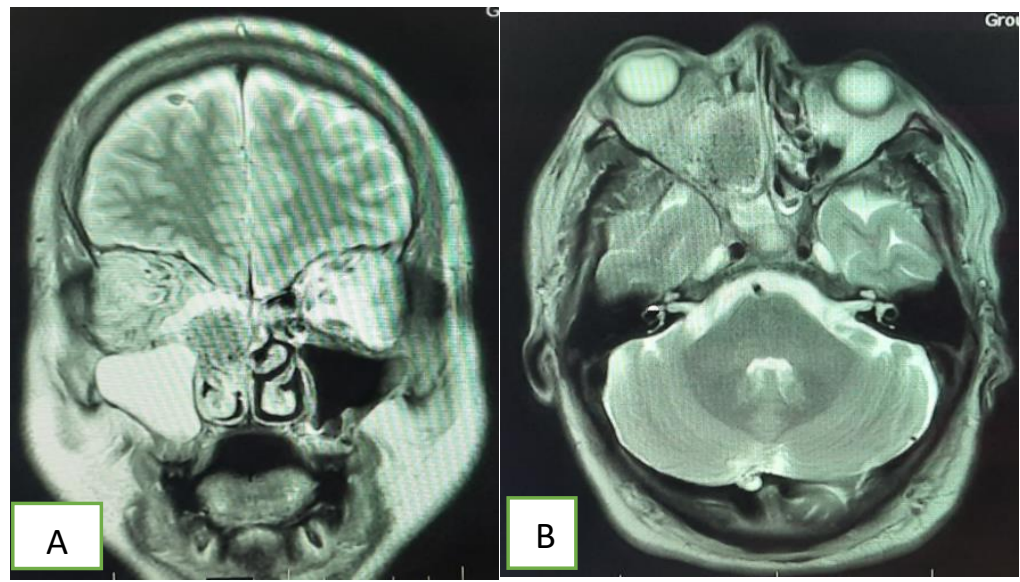

Figure 6: Plain MRI brain and orbit (A:coronal; B:axial) showing features of fungal sinusitis predominantly involving the right side sinuses. There is proptosis of the right eye with fat stranding up to the orbital apex. 


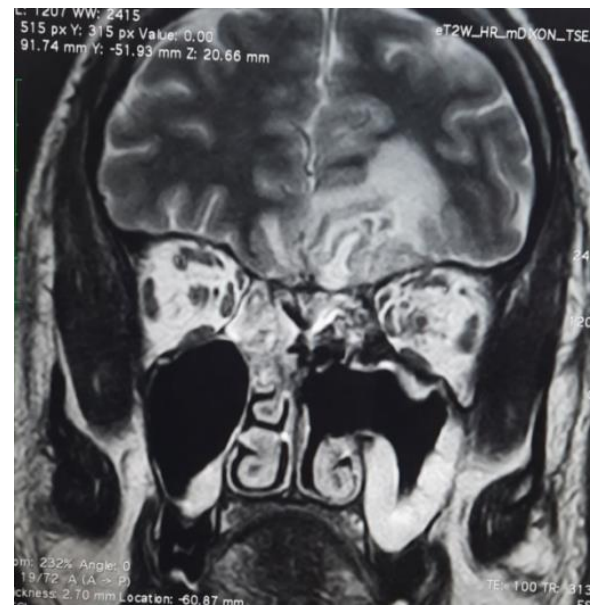

Figure 7: T2 MRI coronal cut of brain and orbits showing postoperative sinus changes with intracranial extension of the disease through cribriform plate.

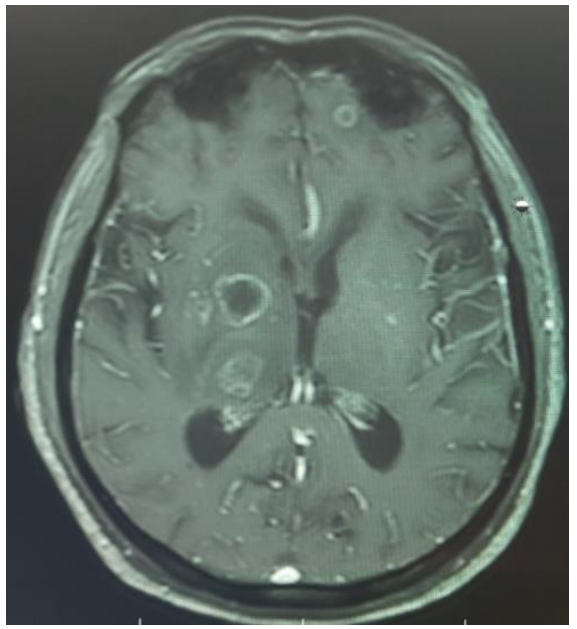

Figure 8: CEMRI axial cut of brain showing multiple predominantly rim enhancing thick walled lesions with surrounding oedema in left frontal, right thalamus and right ganglio-capsular regions.

Among 9 patients of cerebral involvement (rhino-orbital-cerebral mucormycosis), 3 showed cavernous sinus involvement, 4 developed cerebral abscess and 2 patients showed isolated intracranial fungal lesion (likely hematogenous spread). [Figure 9 to 13] All of them landed in ICU during the management and the general condition of 8 patients deteriorated subsequently during ICU care despite appropriate antifungal therapy and did not survive. The only patient who survived had cavernous sinus involvement and underwent orbital exenteration.

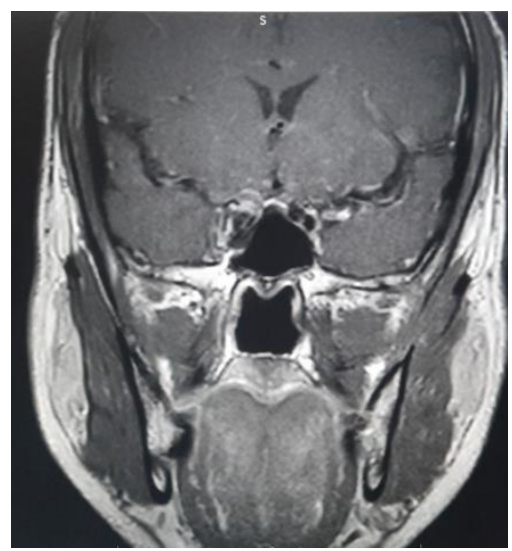

Figure 9: CEMRI coronal cut of brain showing mild asymmetry with thickening convexity of lateral walls of right cavernous sinus with hypoenhancement suggesting early signs of cavernous sinus thrombosis. 


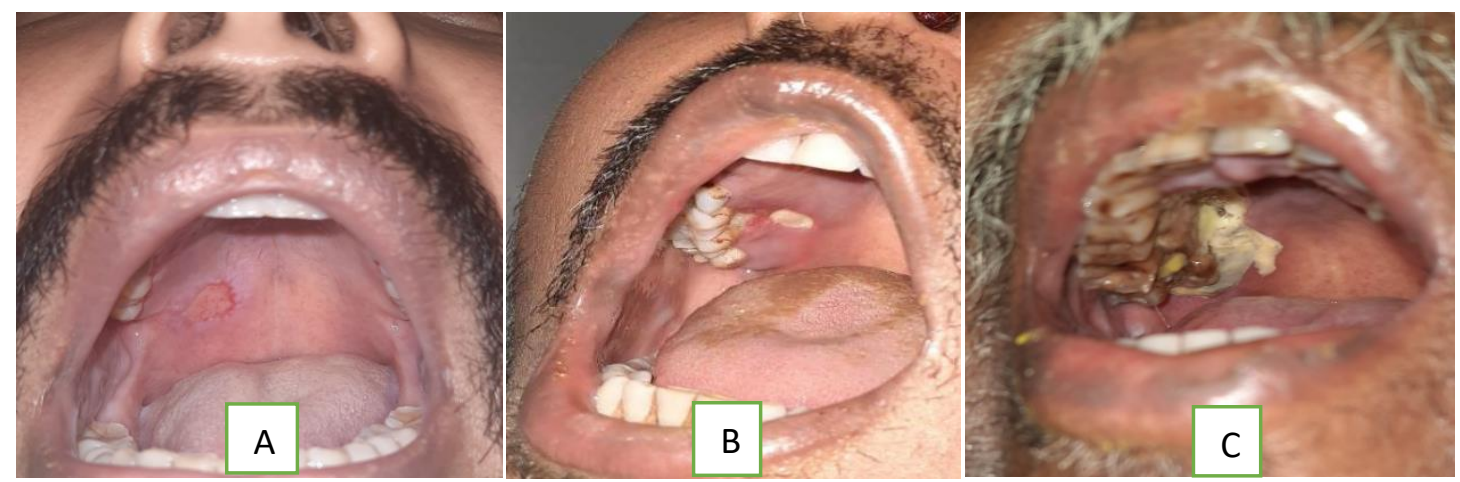

Figure 10: Transoral images showing palatal (floor of right maxiila) involvement due to mucormycosis (in increasing order of severity).

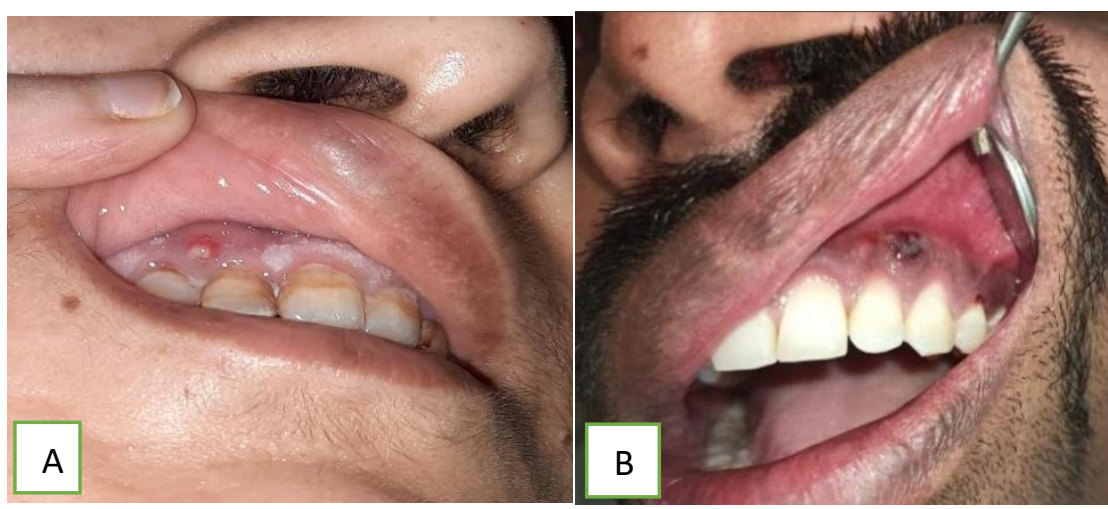

Figure 11: Images showing (A) draining sinus from alveolar process. The patient is having isolated involvement of right maxilla. (B) Showing necrosis of alveolus.

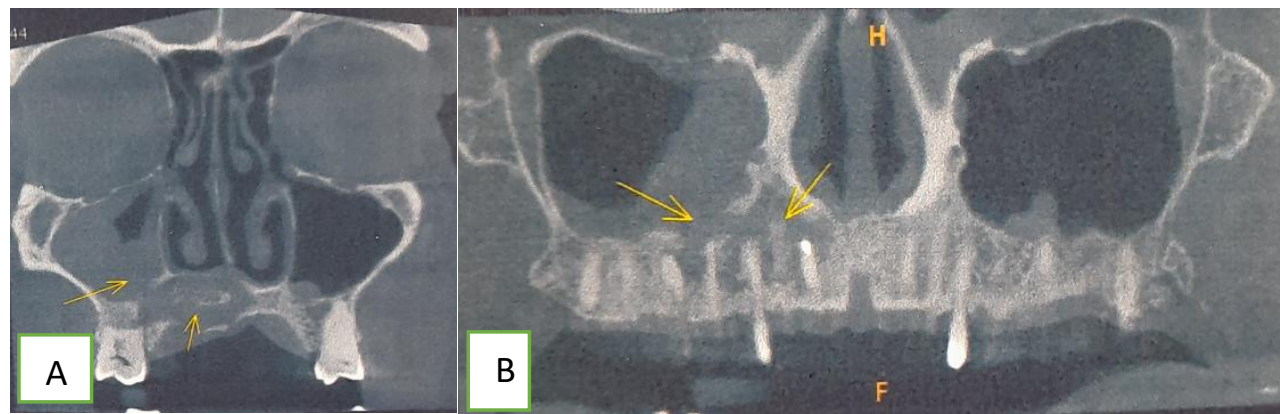

Figure 12: Plain ct scan (coronal) showing erosion of maxilla and alveolus (fungal osteomyelitis).

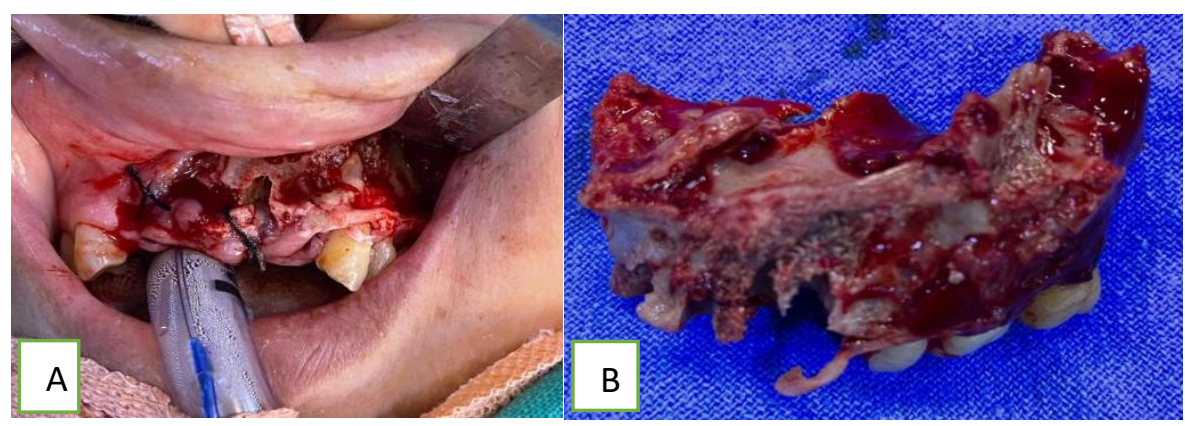

Figure 13: (A)showing necrosed and eroded upper alveolus and (B) showing the same after sequestrectomy. 


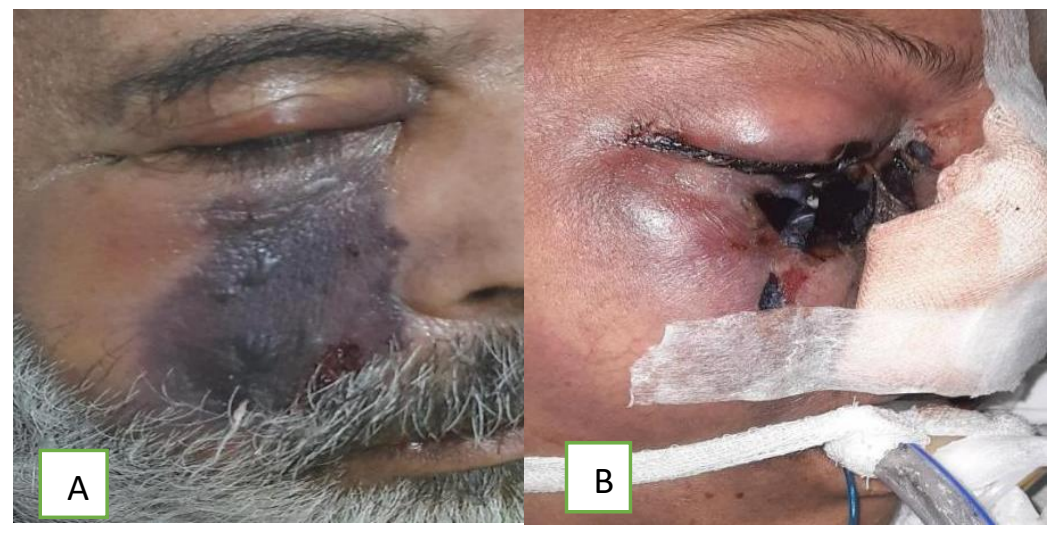

Figure 14: Images showing cutaneous involvement due to mucormycosis. Both patients (A and B) had orbital and cerebral involvement (rhinoorbital-cerebral mucormycosis) and died during ICU care.

In 6 out of 43 patients of rhino-orbital mucormycosis and 4 out of 9 patients of rhino-orbital-cerebral mucormycosis with no visual potential, the decision of orbital exenteration was taken after sub-optimal response of anti-fungals in 72 to 96 hours. But consent for orbital exenteration was not given by 2 patients and their family. So, total 8 orbital exenterations were done during the second stage surgery after initial endoscopic sinus debridement. It was performed by combination of external eyelid sparing and endoscopic power-assisted technique. In all patients who had total ophthalmoplegia PL (-) (10), was due to direct spread or indirect inflammation of orbital apex. These all orbital exenterated patients had extensive necrotic orbital tissue which showed evidence of fungal elements except in one specimen where no fungal element was seen in histopathology but sinus specimen in previous tissue shown fungal elements. Out of these 8 patients 5 patients (4 rhino-orbital mucormycosis and 1 rhino-orbital-cerebral mucormycosis) did not survive but rest 3 patients ( 2 rhino-orbital mucormycosis and 1 rhinoorbital-cerebral mucormycosis) are doing well in follow up. Rest 37 out of 43 rhino-orbital mucormycosis patients showed improvement in ptosis, propotosis, congestion and vision with anti-fungal therapy. Those 2 patients whose family did not give consent were also having cerebral involvement and died after few days of ICU care.

Amongst systemic anti-fungals, liposomal amphotericin-B $(5 \mathrm{mg} / \mathrm{kg} /$ day $)$ was started in all operated 96 patients with close monitoring of renal parameters. In all histopathology proven mucormycosis, oral posaconazole was started, as $300 \mathrm{mg}$ twice a day on the first day as loading dose followed by $300 \mathrm{mg}$ once daily. Due to overloading of mucormycosis patients, we face a great shortage of liposomal form of amphotericin-B, as a result of which patients were switched to conventional amphotericin-B therapy. Many patients developed acute or chronic renal injury which made us switching them to posaconazole or isavuconazole monotherapy. High blood sugar level was managed by endocrinologist with insulin as per sliding scale. All 27 patients with disease limited to sinuses underwent extended endoscopic sinus surgery and debridement. 4 patients showed residual or new enhancement on serial radiological investigation and underwent revision and further debridement.

Condition of 12 patients deteriorated over the next few days after debridement surgery due to acute respiratory distress syndrome and were shifted to non-invasive ventilation whereas 11 of 12 patients got intubated and later on switched to mechanical ventilation and ionotrope support. All 11 patients who were on ventilator support did not survive. Thromboembolic event and cardiac arrest was suspected in all of them.
13 patients (excluding those who required exenteration during second surgery) required revision and further debridement of sinonasal tissue, including those (7 patients) who were operated at some other centres previously.

A different pattern of presentation has been encountered amongst the patients who presented during later phase of this post-COVID-19 mucormycosis. These patients had locally restricted disease to unilateral sinuses and maxilla (palate) [Figure 14 to 17]. 7 patients (out of 27 rhinomucormycosis patients) presented with floor of maxilla and sinus involvement with blackish discoloration within nose or palate, loosening of teeth and abscess in the gingival tissue. They had history of COVID19 infection of more than 3 months, all were diabetics and had history of corticosteroid treatment. All the patients with maxillary involvement had a common clinical presentation of draining sinuses and mobility of teeth, for which routine dental treatment was given. Few had history of multiple consultations of dentists for tooth pain. Hence, the delay in definitive clinical management of mucormycosis. They all showed sequestrum and erosions in maxillary floor and sinusitis in MRI. None had orbital or intracranial involvement. Later, all these patients underwent sequestrectomy and debridement of the infected area along with extraction of involved teeth with the guidance of maxillo-facial surgeon.[Figure 18] Primary closure was achieved in all patients. All the patients with maxillofacial involvement were subjected to amphotericin $\mathrm{B}$ and posaconazole therapy and have not reported any complications and are doing good on follow up.

Interestingly, 3 patients (out of this case series) reported with exclusive involvement of mandible with no signs of disease in maxilla. The presentation in mandible was that of mobile teeth and draining sinuses, possibly suggesting hematogenous spread. The clinical expression of mandibular involvement was classically similar to osteomyelitic process, wherein, the superior alveolar bone was necrotic and easily separable from the underlying basal bone.however, the basal bone was also found to be avascular, necessitating the need to make multiple bur holes for neovascularization.

During this course of time, 14 mortality occurred accounting $18.18 \%$ of mortality rate. All 14 patients who died had thromboembolic events, cardiac arrest and respiratory failure (acute respiratory distress syndrome, ARDS). Most of the patients were discharged after completion of 10 to 14 days of amphoterin-B therapy. They were discharged with oral posaconazole $300 \mathrm{mg}$ once a day for 3 months and were called for regular sino-nasal endoscopic examination. [Table]

\begin{tabular}{|c|c|}
\hline Parameter & Value \\
\hline - Clinically and radiologically suspected cases operated & 96 \\
\hline - Histopathological diagnosed cases (mucormycosis) & 79 \\
\hline $\begin{array}{l}-\quad \text { Age (range in years) } \\
\text { Mean age (in years)[standard deviation] }\end{array}$ & $\begin{array}{l}21-72 \\
52.24[12.23]\end{array}$ \\
\hline - Male:Female & $3.6: 1$ \\
\hline - $\quad$ Patients with active COVID-19 infection & 15 \\
\hline
\end{tabular}




\begin{tabular}{|c|c|}
\hline Patients with post COVID-19 status & 64 \\
\hline \multicolumn{2}{|l|}{ - Clinical category of mucormycosis } \\
\hline & 27 \\
\hline 1. Rhinomucormycosis & 7 \\
\hline With palatal and dental involvement & 43 \\
\hline 2. Rhino-orbital mucormycosis & 11 \\
\hline With PL positive only & 26 \\
\hline With PL negative (total ophthalmoplegia)*, 2 patients had pulmonary mucormycosis also & \\
\hline Vision better than PL positive & 9 \\
\hline & 4 \\
\hline 3. Rhino-orbital-cerebral mucormycosis & 3 \\
\hline With PL negative and total ophthalmoplegia* & \\
\hline With cutaneous involvement [Figure 19] & $52(43+9)$ \\
\hline $\begin{array}{l}\text { Note: Total patients with orbital involvement } \\
\text { Total patients with ophthalmoplegia and PL(-) }\end{array}$ & $10(6+4)$ \\
\hline Known diabetics & 67 \\
\hline Steroid induced diabetes & 12 \\
\hline - $\quad$ Post renal transplant patients (all are with associated diabetes/steroid induced hyperglycemia) & 5 \\
\hline Other comorbidity & \\
\hline Chronic kidney disease (CKD), excluding renal transplant recepients & 6 \\
\hline Chronic liver disease (CLD) & 1 \\
\hline Malignancy & $1 * *$ \\
\hline Heart disease ${ }^{* * *}$ & 4 \\
\hline Hypertension & 14 \\
\hline - Patient with history of ICU care and mechanical ventilation & 18 \\
\hline Patients with history of oxygen therapy & 59 \\
\hline Patients with no history of oxygen therapy & 20 \\
\hline - $\quad$ Isolated Mucor infection ${ }^{\#}$ & 53 \\
\hline Mixed infection (Mucor+Aspergillus+/-Candida) ${ }^{\#}$ & 16 \\
\hline Isolated Aspergillus infection ${ }^{\#}$ & 10 \\
\hline 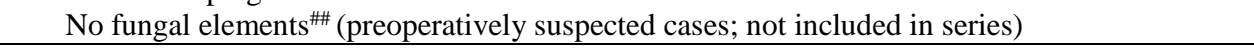 & 17 \\
\hline Patients required revision sinus debridement (including 7 patients referred from other centres) & 13 \\
\hline $\begin{array}{l}\text { Total orbital exenteration performed } \\
\text { Rhino-orbital mucormycosis (died) } \\
\text { Rhino-orbital-cerebral mucormycosis }\end{array}$ & $\begin{array}{l}8 \\
6(4) \\
2(1)\end{array}$ \\
\hline $\begin{array}{l}\text { Outcome (status till } 30^{\text {th }} \text { September 2021) } \\
\text { Alive } \\
\text { Deceased } \\
\text { Loss to follow up (leave against medical advice; LAMA) }\end{array}$ & $\begin{array}{l}63 \\
14(18.18 \%)^{@} \\
2\end{array}$ \\
\hline $\begin{array}{l}\text { *Includes } 1 \text { patient with bilateral total ophthalmoplegia } \\
\text { **Malignant melanoma } \\
\text { ***ischemic heart disease,coronary artery disease, post CABG (coronary artery bypass grafting) } \\
\text { \#Antifungal therapy continued after definitive diagnosis } \\
\text { \#\#Antifungal therapy modified or stopped as per clinical and radiological judgement } \\
{ }^{\circledR} \text { Calculated from } 77 \text { patients, excluding those } 2 \text { patients who went LAMA }\end{array}$ & \\
\hline
\end{tabular}

\section{Discussion}

Mucorales are the moulds found abundantly in the environment, predominantly in hot and humid conditions of tropical countries like India. Rhizopus and Mucor are the two most common species causing mucormycosis. The prevalence of mucormycosis in India is attributed around 140 cases per million population [12]. As mucormycosis is considered a 'diabetes-defining illness', a major bulk is seen among diabetic patients and its aggressiveness is seen more prevalent among uncontrolled diabetics. Rhino-orbital-cerebral mucormycosis is a serious invasive fungal disease, the mortality rate of which is very high even with best treatment. It acts by invading blood vessels and mycotic thrombosis causing infarction and ischemic necrosis of host tissue including surrounding bones [13, 14]. Mucormycosis has put a significant burden over medical system of India and emerged as life threatening complication of COVID-19.

Several factors are considered responsible for this sudden spike in incidence of invasive fungal infection. SARS-CoV-2 infection and post COVID-19 sepsis results in dysregulated and altered immune response causing cytokine storm, thromboembolic events and secondary bacterial

\section{ata of the case serie}

and fungal infections [15]. There are some pathophysiological phenomenons like immune dysregulation, reduced CD4+ T and CD8+T cells and impaired phagocytic immune-cell response that may enhance the risk of invasive fungal infections $[16,17]$. Further the prolonged corticosteroid treatment in such patients especially with pre-existing conditions such as diabetes mellitus, organ transplant, neutropenia and high free iron levels made them a susceptible host for invasive fungal infections like mucormycosis, aspergillosis, cryptococcosis and candidiasis [18]. Corticosteroid induces immunosuppression, lymphopenia and hyperglycemia predisposing to invasive fungal infection [19]. Another indirect potential correlation has been established between COVID-19 and mucormycosis in India, the use of contaminated water with fungal spores for oxygen humidifier or use of contaminated and poor quality industrial oxygen during the shortfall of medical oxygen during the second wave of COVID-19 Infection.

Invasive mucormycosis has not only been seen in severe cases but also in mild and moderate cases of SARS-CoV-2 infections. These patients found to have uncontrolled hyperglycemia, or steroid induced hyperglycemia and on immunosuppresants (renal recipients). Low dose, short duration of corticosteroids has shown benefit in patients of 
moderate to severe illness. But in second wave of SARS-CoV-2 infection higher doses and longer duration of corticosteroids have been used in many patients with severe diseases. The invasive fungal infections in such patients in itself alters the natural history of disease resulting in poor prognosis $[20,21]$. Uncontrolled diabetes and persistent hyperglycemia is considered to effect neutrophil function and hence phagocytosis. Furthermore, diabetic acidosis impairs binding of iron to transferrin letting high free iron promoting fungal growth [22]. These fungi causing invasive infection thrive best in individuals having high serum glucose and acidic condition [23]. It is pertinent to note that majority of invasive fungal infections develop during the later stage of COVID-19 infection. Similar pattern was also noted by Song et al in their study [21].

The fungal spores reach sinuses through inhalation via nares, depositing in nasal mucosa and reaching orbit through lamina papyraceae, ethmoid bone, inferior orbital fissure or via orbital apex. Brain is involved when fungal infection directly involves cribriform plate, supraorbital fissure or by perineural invasion and hematagenous spread [24]. These patients can present with unilateral facial swelling, numbness, proptosis, diminished vision, palatal involvement and headache. The intracranial extension can be in the form of cavernous sinus thrombosis, cerebral infarction, aneurysm or abscess formation. These patients can present with confusion and disorientation. Majority of the patients came into medical attention when they develop some orbital signs and symptoms such as orbital swelling, blurred or double vision, visual loss or ophthalmoplegia.

The nasal endoscopy of suspected patients may show necrosis or discoloration of nasal mucosa with blackish crusting, with or without anesthesia of nasal mucosa. The different diagnostic methods for mucormycosis include $\mathrm{KOH}$ fungal mount, biopsy, fungal staining (calcofluor) and culture. $\mathrm{KOH}$ is a rapid test which shows the presence of broad aseptate filamentous hyphae with right angled irregular branching. Computed tomography $(\mathrm{CT}) /$ magnetic resonance imaging(MRI) of paranasal sinuses, orbits and brain should be obtained to see the extent of the disease. CT may show bony erosion or soft tissue invasion but MRI is more sensitive for detecting characteristic paranasal sinus, orbital and intracranial invasion. MRI may reveal non-enhancing mucosal tissue of sinuses and turbinates, subcutaneous facial tissue infratemporal and temporal fossa inflammatory infiltration. Orbital involvement can be depicted as thickening of medial rectus, proptosis, preseptal edema or orbital apex infiltration. Culture takes many days to grow fungus, but biopsy of the suspected tissue is the mainstay method of definitive diagnosis. Detection of fungal elements can be enhanced by using special fungal stains such as calcofluor white.

Treatment of mucormycosis includes surgical drainage of paranasal sinuses, debridement of necrotic and unhealthy tissues, intravenous antifungal therapy and treating underlying predisposing factors. Hyperbaric oxygen therapy can be added to the regimen but no controlled studies have shown it effective. Early surgical debridement of nasal, paranasal sinus and orbital tissue along with systemic antifungal therapy is the mainstay of treatment of mucormycosis [25].

According to few studies, orbital exenteration is a crucial part of management in extensively diseased orbit, preventing further deterioration [26, 27].But in a retrospective analysis a high mortality rate $(88.9 \%)$ had been seen in patients who underwent exenteration. Deciding the need and timing of orbital exenteration is quite complicated. In limited involvement of orbit with preserved vision, resection of lamina papyracea should be considered. If disease progresses after 72 hours of aggressive antifungal therapy and in non-reactive pupil or no visual perception, orbital exenteration should be done without any further delay.

\section{References}

1. Soman R, Sunavala A. Post COVID-19 mucormycosis-from the frying Pan into the fire. The Journal of the Association of Physicians of India. 2021 Jan;69(1):13-4.
Among anti-fungal agents, amphotericin- $\mathrm{B}$ is the standard antifungal used against invasive mucormycosis. Lipid complex amphotericin-B is well known for its nephrotoxicity which is the major dose-limiting toxicity of using it. Liposomal amphotericin-B is less or non nephrotoxic form of amphotericin-B. Its standard dose is 5 to $10 \mathrm{mg} / \mathrm{kg} /$ day. Other labelled antifungal against invasive mucormycosis used are triazoles like posaconazole and isavuconazole, for atleast 6 weeks. Posaconazole and isavuconazole are the good option as salvage therapy in patients of chronic kidney disease (CKD) or in patients who may develop renal failure with uncontrolled diabetes. These can be used as monotherapy but in refractory cases are usually given as combined therapy. Posaconazole is given twice a day $(300 \mathrm{mg}$ ) orally for one day followed by $300 \mathrm{mg}$ once a day upto 6 months. Similarly isavuconazole is given thrice daily (200mg) orally for 1 day followed by $200 \mathrm{mg}$ once a day upto 6 months. They are used as step-down therapy after initial intravenous amphotericin-B therapy.

Serial radiological investigation (preferably MRI brain, orbit and PNS) is the part of management and is a must to assess the progression or response to therapy. Some studies concluded PET-CT as a useful investigation for postoperative assessment, but cost is the limiting factor [28-30].

The quick identification and diagnosis of invasive fungal infection, subsequently early surgical debridement is the essential management modality in reducing the disease burden and can significantly reduce the morbidity and mortality. Surgical debridement helps in penetration of intravenous antifungal agents more efficiently and limits further spread of disease. Correction of the underlying metabolic and immune derangement is equally important for favorable outcome.

Mucormycosis is a fatal oppurtunistic invasive fungal infection, the prognosis of which is poor affecting mainly immunocompromised and patients with uncontrolled diabetes. The mortality rate for rhino-orbitalcerebral mucormycosis is about 40 to $80 \%$ depending upon the extent of disease [25].

\section{Conclusion}

COVID-19 in diabetics and in immunocompromised patients is associated with various manifestations amongst which mucormycosis is one of the grave association. Further the use of corticosteroids in COVID19 treatment exaggerates the probability of developing invasive fungal infection. Delay in treatment even of few days can increase the mortality. Timely diagnosis and intervention in the form of surgical debridement and anti-fungal therapy are critical aspects in improving clinical outcomes in such patients. The clinicians should now be cautious of possibility of development of invasive fungal infection during the management of covid-19 patients with risk factors and continuous vigilance with regular follow-up in these recovered patients should be strictly followed.

\section{Acknowledgements}

Department of ENT and Head and Neck Surgery, Dental and Maxiloofacial Surgery, Pathology, Microbiology and Radiology, Indraprastha Apollo Hospitals, New Delhi, India.

\section{Conflict of interest}

None.

2. Chen N, Zhou M, Dong X, Qu J, Gong F, Han Y, Qiu Y, Wang J, Liu Y, Wei Y, Yu T. Epidemiological and clinical characteristics of 99 cases of 2019 novel coronavirus pneumonia in Wuhan, China: a descriptive study. The lancet. 2020 Feb 15;395(10223):507-13. 
3. John TM, Jacob CN, Kontoyiannis DP. When uncontrolled diabetes mellitus and severe COVID-19 converge: the perfect storm for mucormycosis. Journal of Fungi. 2021 Apr;7(4):298

4. Lim S, Bae JH, Kwon HS, Nauck MA. COVID-19 and diabetes mellitus: from pathophysiology to clinical management. Nature Reviews Endocrinology. 2021 Jan;17(1):11-30.

5. Papadogeorgakis N, Parara E, Petsinis V, Vourlakou C. A case of successfully treated rhinocerebral mucormycosis: Dental implications. International journal of dentistry. 2010 Jan $1 ; 2010$.

6. Fouad YA, Abdelaziz TT, Askoura A, Saleh MI, Mahmoud MS, Ashour DM, Ashour MM. Spike in rhino-orbital-cerebral mucormycosis cases presenting to a tertiary care center during the COVID-19 pandemic. Frontiers in Medicine. 2021;8.

7. Buil JB, van Zanten AR, Bentvelsen RG, Rijpstra TA, Goorhuis B, van der Voort S, Wammes LJ, Janson JA, Melchers M, Heusinkveld M, Melchers WJ. Case series of four secondary mucormycosis infections in COVID-19 patients, the Netherlands, December 2020 to May 2021. Eurosurveillance. 2021 Jun 10;26(23):2100510.

8. Nehara HR, Puri I, Singhal V, Sunil IH, Bishnoi BR, Sirohi P. Rhinocerebral mucormycosis in COVID-19 patient with diabetes a deadly trio: Case series from the north-western part of India. Indian Journal of Medical Microbiology. 2021 May 26.

9. John TM, Jacob CN, Kontoyiannis DP. When uncontrolled diabetes mellitus and severe COVID-19 converge: the perfect storm for mucormycosis. Journal of Fungi. 2021 Apr;7(4):298.

10. Sharma S, Grover M, Bhargava S, Samdani S, Kataria T. Post coronavirus disease mucormycosis: a deadly addition to the pandemic spectrum. The Journal of Laryngology \& Otology. 2021 May;135(5):442-7.

11. Moorthy A, Gaikwad R, Krishna S, Hegde R, Tripathi KK, Kale PG, Rao PS, Haldipur D, Bonanthaya K. SARS-CoV-2, Uncontrolled Diabetes and Corticosteroids-An Unholy Trinity in Invasive Fungal Infections of the Maxillofacial Region? A Retrospective, Multi-centric Analysis. Journal of maxillofacial and oral surgery. 2021 Mar 6:1-8.

12. Prakash H, Chakrabarti A. Global epidemiology of mucormycosis. Journal of Fungi. 2019 Mar;5(1):26.

13. Werthman-Ehrenreich A. Mucormycosis with orbital compartment syndrome in a patient with COVID-19. The American journal of emergency medicine. 2021 Apr 1;42:264e5.

14. Petrikkos G, Skiada A, Lortholary O, Roilides E, Walsh TJ, Kontoyiannis DP. Epidemiology and clinical manifestations of mucormycosis. Clinical Infectious Diseases. 2012 Feb 1;54(suppl_1):S23-34.

15. Mehta S, Pandey A. Rhino-orbital mucormycosis associated with COVID-19. Cureus. 2020 Sep;12(9).

16. Gangneux JP, Bougnoux ME, Dannaoui E, Cornet M, Zahar JR. Invasive fungal diseases during COVID-19: We should be prepared. Journal de mycologie medicale. 2020 Jun;30(2):100971.

17. Spellberg B, Edwards Jr J, Ibrahim A. Novel perspectives on mucormycosis: pathophysiology, presentation, and management. Clinical microbiology reviews. 2005 Jul;18(3):556-69.
18. Song G, Liang G, Liu W. Fungal co-infections associated with global COVID-19 pandemic: a clinical and diagnostic perspective from China. Mycopathologia. 2020 Jul 31:1-8.

19. Ibrahim AS, Spellberg B, Walsh TJ, Kontoyiannis DP. Pathogenesis of mucormycosis. Clinical Infectious Diseases. 2012 Feb 1;54(suppl_1):S16-22.

20. Nasir N, Farooqi J, Mahmood SF, Jabeen K. COVID-19associated pulmonary aspergillosis (CAPA) in patients admitted with severe COVID-19 pneumonia: An observational study from Pakistan. Mycoses. 2020 Aug;63(8):766-70.

21. Bartoletti M, Pascale R, Cricca M, Rinaldi M, Maccaro A, Bussini L, Fornaro G, Tonetti T, Pizzilli G, Francalanci E, Giuntoli L. Epidemiology of invasive pulmonary aspergillosis among COVID-19 intubated patients: a prospective study. Clinical Infectious Diseases. 2020 Jul 28.

22. Balai E, Mummadi S, Jolly K, Darr A, Aldeerawi H. Rhinocerebral mucormycosis: a ten-year single centre case series. Cureus. 2020 Nov;12(11).

23. Gale GR, Welch AM. Studies of opportunistic fungi. I. Inhibition of Rhizopus oryzae by human serum. American Journal of Medical Sciences. 1961;241(5):604-12.

24. Sen M, Lahane S, Lahane TP, Parekh R, Honavar SG. Mucor in a viral land: a tale of two pathogens. Indian journal of ophthalmology. $2021 \mathrm{Feb} ; 69(2): 244$.

25. Cornely OA, Alastruey-Izquierdo A, Arenz D, Chen SC, Dannaoui E, Hochhegger B, Hoenigl M, Jensen HE, Lagrou K, Lewis RE, Mellinghoff SC. Global guideline for the diagnosis and management of mucormycosis: an initiative of the European Confederation of Medical Mycology in cooperation with the Mycoses Study Group Education and Research Consortium. The Lancet infectious diseases. 2019 Dec 1;19(12):e405-21.

26. Songu M, Unlu HH, Gunhan K, Ilker SS, Nese N. Orbital exenteration: A dilemma in mucormycosis presented with orbital apex syndrome. American journal of rhinology. 2008 Jan;22(1):98-103.

27. Pelton RW, Peterson EA, Patel BC, Davis K. Successful treatment of rhino-orbital mucormycosis without exenteration: the use of multiple treatment modalities. Ophthalmic Plastic \& Reconstructive Surgery. 2001 Jan 1;17(1):62-6.

28. Mukherjee B, Raichura ND, Alam MS. Fungal infections of the orbit. Indian journal of ophthalmology. 2016 May;64(5):337.

29. Liu Y, Wu H, Huang F, Fan Z, Xu B. Utility of 18 F-FDG $\mathrm{PET} / \mathrm{CT}$ in diagnosis and management of mucormycosis. Clinical nuclear medicine. 2013 Sep 1;38(9):e370-1.

30. Altini C, Ferrari C, Rubini D, Dicuonzo F, Rubini G. (18) FFDG PET/CT contribution to diagnosis and treatment response of rhino-orbital-cerebral mucormycosis. Hellenic journal of nuclear medicine. 2015 Jan 1;18(1):68-70. 